\title{
Authenticity, and the Stimulated Self: Neurosurgery for Anorexia Nervosa
}

\author{
Hannah Maslen, Jonathan Pugh and Julian Savulescu
}

Mueller et al. address a range of ethical considerations associated with neurosurgical interventions for the treatment of Anorexia Nervosa (AN), arguing for several protective measures to safeguard clinical research and practice. This is an important article, which provides a thorough review of current neurosurgical research and presents key insights into challenges associated with compromised decision-making capacities in the context of AN and the early average age of onset.

We have argued elsewhere that further considerations relate to the ways in which the potential mechanisms deep brain stimulation (DBS) affect the patient's desires, cognitive control and emotions, with distinct consequences for autonomy (these points are developed in Maslen et al. (under review)). In addition, as we will outline here, ethical analysis of DBS and ablative surgery for AN should explicitly address the authenticity of patients' desires and experiences.

Mueller et al. do consider the related issues of personality and identity. First, they suggest that, since it is not possible to adequately inform patients about the effects that psychiatric neurosurgery might have on their personality or identity, it might not be possible for patients to provide sufficiently informed consent to the procedure (17). Second, they point out that DBS might alter personality or identity when considering coercive interventions in adolescents, concluding that this constitutes one of two jointly-decisive reasons not to perform neurosurgery against the adolescent's will. In this context, they hint at authenticity when they quote Charland's claim that 'many AN patients oscillate ambiguously between the view that anorexia is a part of their true self, and that it has completely taken over their self'(x).

However, it is somewhat striking that they neither use nor examine the concept of authenticity. We describe the way this concept features in discussions of AN, and suggest that a deeper understanding of authenticity reveals further ethical problems that Mueller et al. do not consider. In this commentary, we focus on DBS as the neurosurgical intervention about which there is the most optimism in the context of AN treatment. We note that ablative surgery will raise other profound issues because they will not be reversible.

\section{Authenticity and Anorexia Nervosa}

In general terms, authenticity is understood to amount to living in accordance with one's 'true self', and it is often thought to be of intrinsic value. The concept of authenticity is distinct from that of personal identity. According to widely-endorsed psychological accounts of personal identity, in order for an agent to maintain their identity over time, they must retain psychological continuity and connectedness, (Parfit 1984). With this in mind, a neuro-intervention could plausibly have inauthentic effects without threatening the agent's identity. This would be the case if the recipient retained a sufficient number of psychological connections (such as memories and values) following the intervention, but these effects were not deemed to be true to her 'real self'. Conversely, an intervention might threaten an agent's personal identity if it severed a sufficient number of her psychological connections, but we could claim that the effects of the intervention are authentic to the (different) agent who now exists. 
Within the literature on AN, determining how authenticity should be conceived has proven to be complex. Interviews conducted by Hope et al. (2011) reveal that different patients have different experiences of the authenticity of self, desires and choices. Experiences can also change over the course of an individual patient's illness and recovery: the anorexic patient at some points might experience her anorexic traits as inauthentic and seek to extirpate them; at other times she might view her anorexic parts as authentic, but significantly problematic and in need of management; other times she might embrace her anorexic parts as valuably authentic, seeking to promote rather than minimize them.

\section{Implications for patient decision-making}

DBS treatment, which has the potential to reversibly modulate desires, cognitive control and affect, complicates considerations of authenticity further. To illustrate, suppose that an anorexic patient validly consents to undergoing DBS. Suppose that, while experiencing the effects of the stimulation, she embraces the changes to her desires precipitated by the stimulation, telling her care team she endorses them and would like to continue stimulation in the future. However, when not stimulated, she laments the changes that occurred whilst undergoing stimulation, and withdraws her consent to continue treatment. ${ }^{1}$

This possibility adds a further layer of uncertainty to existing difficulties in determining the authentic wishes of the anorexic patient. If, as qualitative studies suggest, the experience of AN tends towards frequent changes in beliefs and preferences, and even profound ambivalence at one time (see Hope et al. 2013), the possibility of adding a further 'self' - the 'stimulated self' - renders determination of which preferences are authentic even more difficult; which the patient identifies with when she is under the influence of DBS and when stimulation ceases might conflict. Assuming that the patient retains a sufficient degree of psychological continuity and connectedness over this period of time though, we may still coherently speak of her being the same person. This then, is a problem of authenticity, rather than of identity.

In such cases, it might be argued that we should respect the wish expressed under the influence of treatment, since the desires that undergird the patient's refusal of treatment have a 'pathological origin', thus failing to reflect what the agent authentically desires. For instance, Tan and colleagues have suggested that assigning paramount importance to thinness can be understood to be pathological to the extent that this sort of evaluation satisfies the diagnostic criteria of AN (Tan et al. 2007, 278).

However, this claim requires further support. We note, with Craigie (2011), that Tan et al.'s proposal is somewhat question-begging; the inclusion of this sort of evaluation in the diagnostic criteria of AN itself seems to presuppose that the evaluation is pathological (Craigie 2011). Without further justification, physicians should not claim that only the desire to continue treatment is authentic. Whilst a patient might express such a desire under the influence of DBS, we should be wary of the profound effects

\footnotetext{
${ }^{1}$ Similar scenarios have been presented elsewhere (Cabrera, et al. 2014).
} 
of stimulation on the patient's drives and emotions. It is possible that the patient may feel alienated by the changes effected by stimulation.

For example, DBS might be used to impose a change on the agent's motivating desires such that a decision to continue stimulation could be inauthentic. Indeed, one proposed mechanism for DBS would involve stimulating the part of the brain involved in reward processing (Park at al. 2014). However, the mere fact that an agent finds something rewarding and pursues it on this basis, does not guarantee that she values or rationally endorses it. Addiction provides an interesting comparison in this regard. Reward can generate strong motivation, and the fact that a person continues to stimulate a part of the brain implicated in reward does not necessarily mean that the choice is an expression of the agent's values or her authentic self.

Thus, it is imperative that clinicians encourage the patient to reflect on the changes both when 'on' and 'off' stimulation to better determine whether the patient embraces them as authentic. The preferences of the stimulated self must not be taken to necessarily have priority just because pathology appears reduced.

\section{Implications for assessing psychiatric improvements}

We further suggest that questions pertaining to authenticity complicate the matter of whether neurosurgery for AN has straightforwardly beneficial effects on psychiatric symptoms in the way that Mueller et al. claim. They say that their data indicate that stereotactic neurosurgery for AN improves psychiatric symptoms (13). However, given the way in which DBS can modulate desires and affect, we cannot tell whether the psychiatric improvements that the patient reports are authentic or not simply by assessing the stimulated patient's apparent psychiatric state. It is a further question still whether inauthentic 'improvements', if present in the stimulated patient, should be classified as improvements, all things considered.

\section{Authenticity and Autonomy}

There are thus authenticity-related concerns regarding the use of DBS to treat AN. However, we have argued elsewhere that different DBS mechanisms will have distinct implications for the patient's capacity to make autonomous decisions (Maslen et al. under review). Briefly, we suggest that a mechanism that increases the patient's capacity for top-down cognitive control over compulsive behaviors would be a paradigm example of an intervention that increases the recipient's capacity for autonomous decision-making. Similarly, a mechanism that modulates affect may promote autonomy by reducing the anxiety which sometimes prevents patients from assigning the correct epistemic weight to facts about their low weight, and from engaging in healthier eating behaviors (Hope et al. 2013). This is not to say that DBS always increases the patient's capacity for autonomous decision-making; in fact, it may be detrimental to to alter bottom-up motivating desires that were not endorsed by the patient at a higher-order level. However, when it seems plausible to say that DBS does increase the patient's capacity for autonomy, clinicians may have to make difficult treatment decisions about whether to prioritize the authenticity or the autonomy of the patient's stimulated self. 


\section{References}

Cabrera, Laura Y., Emily L. Evans, and Roy H. Hamilton. 2014. "Ethics of the Electrified Mind: Defining Issues and Perspectives on the Principled Use of Brain Stimulation in Medical Research and Clinical Care.” Brain Topography 27 (1): 33-45. doi:10.1007/s10548-013-0296-8.

Craigie, Jillian. 2011. "Competence, Practical Rationality and What a Patient Values." Bioethics 25 (6): 326-33. doi:10.1111/j.1467-8519.2009.01793.x.

Hope, Tony, Jacinta Tan, Anne Stewart, and Ray Fitzpatrick. 2011. "Anorexia Nervosa and the Language of Authenticity." Hastings Center Report 41 (6): $19-29$.

Hope, Tony, Jacinta Tan, Anne Stewart, and John McMillan. 2013. “Agency, Ambivalence and Authenticity: The Many Ways in Which Anorexia Nervosa Can Affect Autonomy." International Journal of Law in Context 9 (01): 2036.

Maslen, Hannah, Jonathan Pugh, and Julian Savulescu. (Under review), "The Ethics of Deep Brain Stimulation for the Treatment of Anorexia Nervosa".

Parfit, Derek. 1984. Reasons and Persons. Oxford: Clarendon Press.

Park, Rebecca J., Lauren R. Godier, and Felicity A. Cowdrey. 2014. "Hungry for Reward: How Can Neuroscience Inform the Development of Treatment for Anorexia Nervosa?" Behaviour Research and Therapy 62: 47-59.

Tan, Jacinta, Anne Stewart, Ray Fitzpatrick, and R. A. Hope. 2007. "Competence to Make Treatment Decisions in Anorexia Nervosa: Thinking Processes and Values." Philosophy, Psychiatry, \& Psychology 13 (4). 